\title{
Resistance Spikes at Transitions between Quantum Hall Ferromagnets
}

\author{
E. P. De Poortere, E. Tutuc, S. J. Papadakis, and M. Shayegan \\ Department of Electrical Engineering, Princeton University, Princeton, New Jersey 08544
}

(Dated: November 17, 2018)

\begin{abstract}
We report a manifestation of first-order magnetic transitions in two-dimensional electron systems. This phenomenon occurs in aluminum arsenide quantum wells with sufficiently low carrier densities and appears as a set of hysteretic spikes in the resistance of a sample placed in crossed parallel and perpendicular magnetic fields, each spike occurring at the transition between states with different partial magnetizations. Our experiments thus indicate that the presence of magnetic domains at the transition starkly increases dissipation, an effect also suspected in other ferromagnetic materials. Analysis of the positions of the transition spikes allows us to deduce the change in exchange-correlation energy across the magnetic transition, which in turn will help improve our understanding of metallic ferromagnetism.
\end{abstract}

Ferromagnetism in metallic systems, also known as itinerant electron ferromagnetism, has thus far revealed few of its secrets to scientists. In one of its well-known occurrences, in transition elements $\mathrm{Fe}, \mathrm{Co}$, and $\mathrm{Ni}$, metallic ferromagnetism is believed to stem from a partially filled $3 \mathrm{~d}$ band of electrons with unbalanced spin populations, although the properties of their magnetic moments at nonzero temperature are still unclear (1) despite some recent theoretical progress (2). In dilute electron gases, the appearance of spontaneous magnetization at sufficiently low densities, as evidenced by recent experiments in doped hexaborides (3), is also subject to debate, with the critical density at the ferromagnetic to paramagnetic transition still uncertain (4). One difficulty in modeling these materials lies in their complexity, because itinerant charged carriers in these systems are subject not only to electron-electron interactions, but also to atomic potentials resulting in an intricate density of states or to local moments of other atoms in the material.

Two-dimensional (2D) electron systems in modulationdoped semiconductor heterostructures, on the other hand, provide an ideal system for the study of itinerant electron ferromagnetism, as interactions with their host material are almost entirely contained in the effective mass $\left(m^{*}\right)$ and effective $g$-factor $\left(g^{*}\right)$ of electrons. Given these two renormalizations, carriers in these structures behave as a nearly free electron gas. In a perpendicular magnetic field $\left(B_{\perp}\right), 2 \mathrm{D}$ electrons condense into a ladder of energy levels, called Landau levels, which are separated by the cyclotron energy $\hbar \omega_{c}=\hbar e B_{\perp} / m^{*}$. The total magnetic field $\left(B_{t o t}\right)$ also couples to the electron spin, and leads to an additional (Zeeman) energy $\pm \frac{1}{2}\left|g^{*}\right| \mu_{B} B_{t o t}$ (where $\mu_{B}$ is the Bohr magneton, $e \hbar / 2 m_{e}$, and $m_{e}$ is the bare electron mass), which splits each Landau level into two separate levels. The number of occupied spin-split Landau levels at a given field is called the filling factor, $\nu$. This discrete level structure gives rise to the quantum Hall (QH) effect, the vanishing of longitudinal resistance
$\left(R_{x x}\right)$ and the quantization of the Hall resistance, that occurs when an integral number of Landau levels are occupied. In the setup we use for our experiments (inset of Fig. 1B), where the sample is tilted at angle $\theta$ with respect to the magnetic field, both Zeeman and cyclotron energies can be tuned independently.

Figure 1A depicts the evolution of electronic levels in a constant $B_{\perp}$, as $B_{t o t}($ or $\theta$ ) is increased: The Zeeman splitting increases with $B_{t o t}$, so that in an independentelectron picture, energies cross or come into "coincidence" for particular values of $\theta$. On either side of these coincidences, the magnetization of the electron system thus takes on distinct values, corresponding to QH ferromagnets (or "Ising" states) of various strengths. Figure 1B highlights our main finding, namely that the transitions between these ferromagnets provoke sharp peaks in the magnetoresistance. These new peaks, marked by arrows, are distinct from the usual maxima between $\mathrm{QH}$ minima and are visible here near $\nu=3,5$, and 7 . Moreover, as we show later in this report, the magnitude of the spikes at low temperatures depends strongly on magnetic field sweep direction, a clear indication that the peaks occur at the magnetic phase transition. In addition, we are able to record these peaks for a broad range of filling factors and carrier densities. This allows us to establish a simple model for the field at which the transitions take place and, with this model, to obtain the exchange-correlation energy gained by the system as it makes the transition.

Our results can be compared with tilt experiments performed in various $2 \mathrm{D}$ systems (5-8). These previous measurements either show the expected disappearance of the $\mathrm{QH}$ resistance minimum at coincidence, corresponding to the vanishing of the gap between Landau levels, or, as in $(6,7)$, reveal the persistence of the $R_{x x}$ minimum at coincidence, indicating a more subtle anticrossing of the Landau levels at the Ising transition. Our experiment, on the other hand, displays detailed struc- 

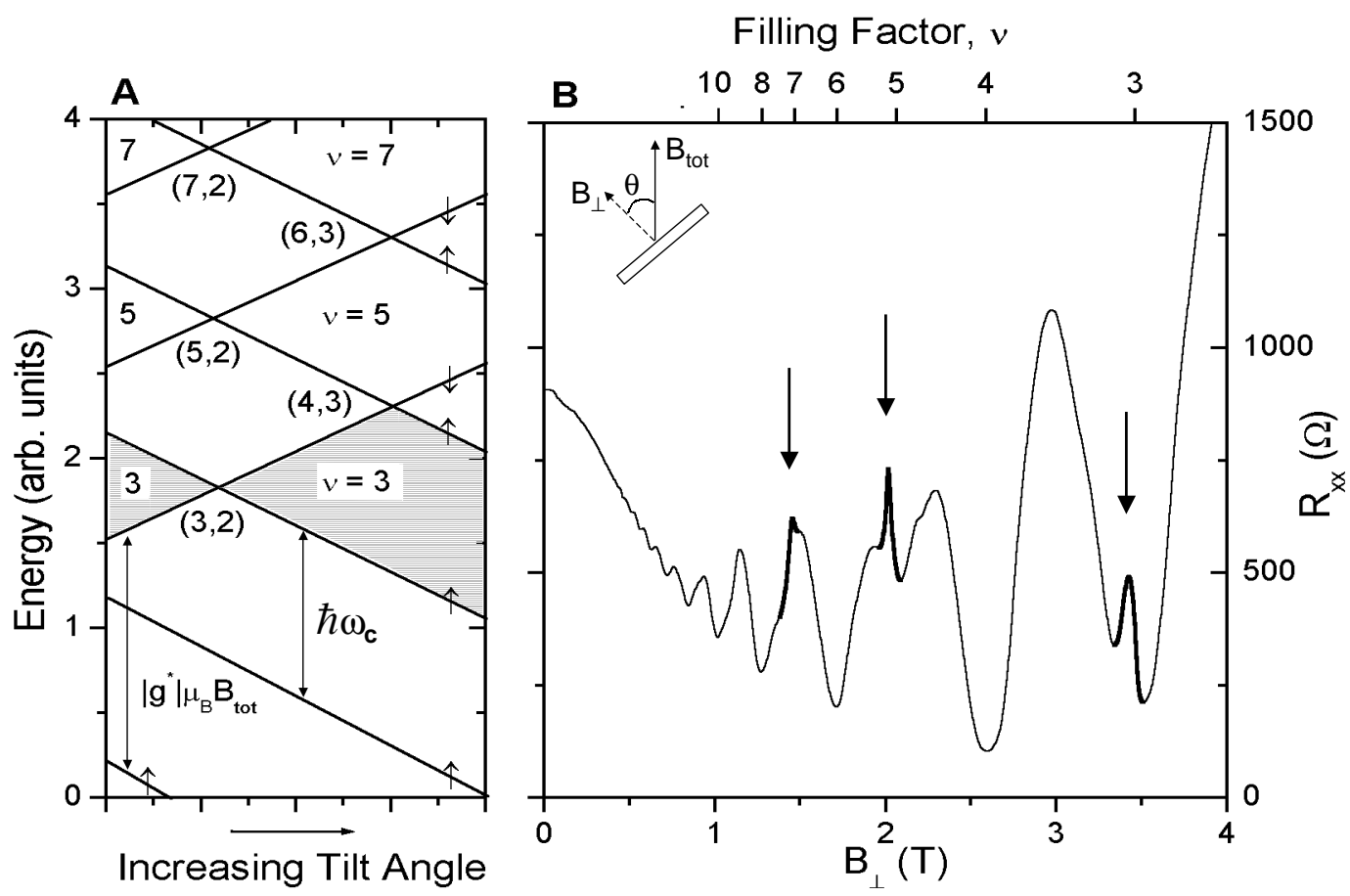

FIG. 1: (A) Diagram of the Landau energy levels in a 2D electron gas, showing the spin-up and spin-down levels intersecting as parallel field (or angle $\theta$ ) is increased. Indices $(\nu, l)$ label each crossing, where $\nu$ maps to the filling factor of the nearest magnetoresistance minimum and $l$ is the number of fully polarized filled levels at the $(\nu, l)$ coincidence. (B) Longitudinal magnetoresistance $\left(R_{x x}\right)$ of the 2 D electron gas in our AlAs quantum well at $\theta=38^{\circ}$ and at $T \approx 300$ $\mathrm{mK}$, showing resistance spikes near filling factors 3,5 and 7 .

ture within the $\mathrm{QH}$ minimum: The latter remains strong over most of its range, except in a small field interval where the resistance rises sharply. Fractional QH states can also undergo a spin-related phase transition (9-12), which appears to share common traits with the integer $\mathrm{QH}$ transition. A theoretical investigation of magnetism and magnetic transitions in the integer $\mathrm{QH}$ regime has been addressed in (13), motivated in part by experiments on pseudospin Ising transitions in wide GaAs quantum wells (the pseudospin of an electron refers to its layer or subband index) $(8,14)$.

The 2D electron gas we study resides in the conduction band of a $150 \AA$-wide AlAs quantum well, bordered by $\mathrm{Al}_{0.39} \mathrm{Ga}_{0.61} \mathrm{As}$ barriers and modulation-doped with a front layer of Si. Our structures were grown by molecular-beam epitaxy over GaAs substrates oriented along various crystal lattice planes, and the data we present are from a sample grown on a (411)B-oriented substrate. We performed magnetotransport measurements in pumped ${ }^{3} \mathrm{He}$ and dilution refrigerators with magnetic fields up to $18 \mathrm{~T}$. Through a combination of sample illumination and back-gate biasing, we were able to vary the carrier density $(n)$ from $1.6 \times 10^{11}$ to $5.2 \times 10^{11}$ $\mathrm{cm}^{-2}$.

Our choice of AlAs as a host material for 2D electrons stems from the magnitude of their effective mass (15) and of their $g$-factor, both of which are substantially larger than in GaAs. The larger mass in AlAs makes carriers more dilute and therefore more sensitive to many-body effects; the larger $g$-factor, equal to 1.9 (16), implies that the Zeeman and cyclotron energies become comparable for a wide range of experimental conditions. Moreover, our samples have electron mobilities as high as $20 \mathrm{~m}^{2} / \mathrm{Vs}$ for $n=5 \times 10^{11} \mathrm{~cm}^{-2}$, and exhibit fractional QH states up to third-order fractions, indicating that many-body effects dominate the energetics of our 2D electron system at low temperatures.

We studied the behavior of the anomalous peaks while changing various parameters: tilt angle, temperature, field sweep direction, and sample density. Fig. 2, A and $\mathrm{B}$, shows the evolution of magnetoresistance as we change the tilt angle of our sample (here $n=2.5 \times 10^{11}$ 

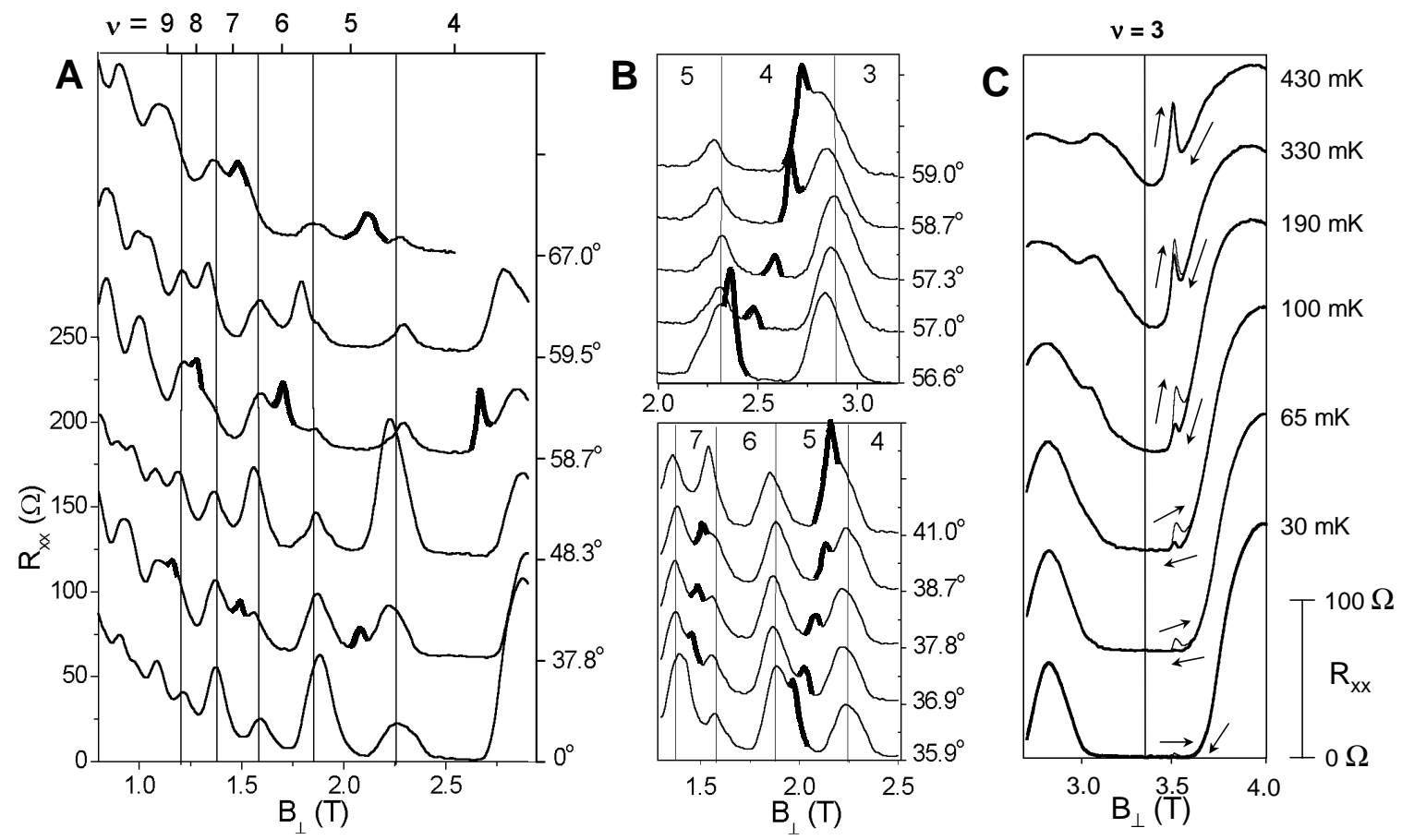

FIG. 2: (A) Magnetoresistance of the 2D electron gas at $n=2.5 \times 10^{11} \mathrm{~cm}^{-2}$ for various tilt angles at $T=30 \mathrm{mK}$. New peaks are seen near $\nu=5,7$, and 9 for $\theta=37.8^{\circ}$ and $\theta=67.0^{\circ}$, and near $\nu=4,6$, and 8 for $\theta=58.7^{\circ} . R_{x x}$ traces are shifted vertically for clarity. Base lines $\left(R_{x x}=0\right)$ are marked by the right tick marks, which indicate the corresponding tilt angle. (B) Movement of the peaks from higher to lower filling as tilt angle increases. (C) Hysteresis and temperature dependence of the magnetoresistance peak near $\nu=3$, at a tilt angle $=37.8^{\circ}$. No hysteresis is seen at $430 \mathrm{mK}$, whereas at lower temperatures the peak becomes increasingly hysteretic. At $30 \mathrm{mK}$, the peak has all but vanished in the downward sweep, whereas it is still observable (in an enlarged trace) in the upward sweep.

$\left.\mathrm{cm}^{-2}\right)$. At three angles, $37.8^{\circ}, 58.7^{\circ}$ and $67.0^{\circ}$, additional peaks appear within the $\mathrm{QH}$ minima. At $\theta=37.8^{\circ}$ and $\theta=67.0^{\circ}$, peaks are seen near odd fillings $\nu=5,7$, and 9 , and for $\theta=58.7^{\circ}$ they occur near even fillings $\nu=4,6$, and 8 . Moreover, as we increase $\theta$ around these special angles (Fig. 2B), we see the peaks travel from low fields to high fields. This allows us to rule out an inhomogeneous density in the sample as a possible explanation for the peaks, because inhomogeneities would not depend on the parallel field.

Evidence that the anomalous peaks correspond to a phase transition is displayed in Fig. 2C: Tuning the 2D electrons to the state where a peak is seen near $\nu=3$, we observe magnetic hysteresis at the precise location of the anomalous peak. At high temperatures $(T=430 \mathrm{mK})$, hysteresis is absent, whereas for $T<330 \mathrm{mK}$, upward and downward sweeps diverge increasingly as temperature is lowered. For $T<65 \mathrm{mK}$ the downsweep peak cannot be discerned, and likewise at $T=30 \mathrm{mK}$, the upsweep peak has nearly vanished.

We now show that the positions of the peaks in $\left(B_{\perp}\right.$, $\left.B_{t o t}\right)$ phase space also lend themselves to a simple interpretation in terms of a phase boundary between ferromagnetic states. We note that, as $\theta$ increases, peaks appear alternatively within odd and even QH states. Following a standard argument used in (5), we can thus put the peaks in a one-to-one correspondence with the levelcrossings of Fig. 1A. We label these crossings with an index $(\nu, l)$, where $\nu$ is the number of filled levels at the nearest $R_{x x}$ minimum and $l$ is the rank of the coinci- 

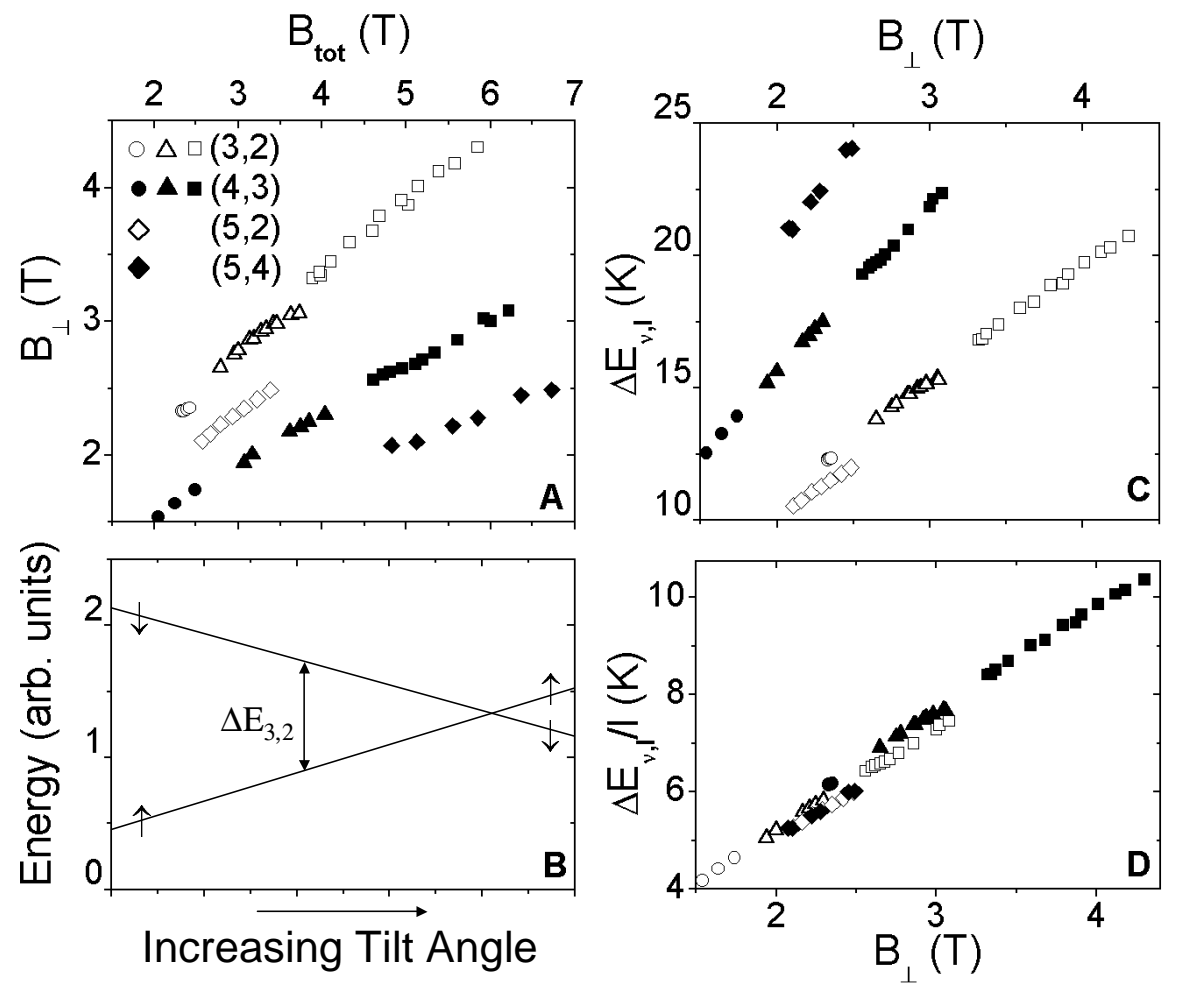

FIG. 3: (A) Positions of the magnetoresistance peaks, plotted in $\left(B_{\perp}, B_{t o t}\right)$ space for various densities (squares/diamonds, $n=2.7 \times 10^{11} \mathrm{~cm}^{-2}$; triangles, $n=2.1 \times 10^{11} \mathrm{~cm}^{-2}$; circles, $\left.n=1.6 \times 10^{11} \mathrm{~cm}^{-2}\right)$. $(\mathbf{B})$ Energy levels close to the $\nu=3$ coincidence, showing the field at which electrons transfer to the spin-up level. At the transition, the energy difference between levels equals $\Delta E_{3,2}$. For clarity, only independent-electron energy levels are drawn. (C) Exchange-correlation energies $\Delta E_{\nu, l}$ extracted from (A) (with same symbols). (D) Scaling of $\Delta E_{\nu, l}$ with $l$, the number of occupied polarized energy levels.

dence, which we define as the number of fully polarized levels below the highest occupied level (which undergoes the transition). In Fig. 3A, we plot the positions of the peaks for different densities and tilt angles (17).

Let us focus first on the $(3,2)$ transition, near $\nu=3$. In an independent-electron picture, where the only energy contributions are from cyclotron and Zeeman energies ("bare" energies), the transition between unpolarized and polarized states happens exactly when the bare energy levels cross. By contrast, as shown in Fig. 3B, interacting electrons may opt to transfer to the unoccupied level before the bare-level coincidence, i.e. at a smaller $\theta$, in order to align their spins with the spins of the electrons in the lower levels [see (7) for a similar argument]. Calling $\Delta E_{3,2}$ the energy difference between bare levels at the $(3,2)$ transition, we can write this difference as
(18)

$\left(\frac{5}{2} \hbar \omega_{c}-\frac{1}{2}\left|g^{*}\right| \mu_{B} B_{t o t}\right)-\left(\frac{1}{2} \hbar \omega_{c}+\frac{1}{2}\left|g^{*}\right| \mu_{B} B_{t o t}\right)=\Delta E_{3,2}$

where $\omega_{c}=e B_{\perp} / m^{*}$. If we now write the total energy $E_{\nu, \pm 1 / 2}$ of electrons of spin $\pm 1 / 2$ in Landau level $\nu$ as a sum of their bare energies and of an exchange-correlation energy term $X_{\nu, \pm 1 / 2}$, we can then interpret $\Delta E_{3,2}$ as the difference $\left(X_{0,-1 / 2}-X_{2,+1 / 2}\right)$, because the transition takes place when the two competing energies $E_{0,-1 / 2}$ and $E_{2,+1 / 2}$ are equal. Using both Eq. 1 and our data in Fig. 3A, we deduce the dependence of $\Delta E_{3,2}$ on $B_{\perp}$ at the transitions, which we plot in Fig. $3 \mathrm{C}$, using $g^{*}=1.9$ and $m^{*}=0.41 m_{e}$. Although no accurate calculations for the exchange-correlation energy terms in $\Delta E_{3,2}$ exist at present, we expect $\Delta E_{3,2}$ to be a fraction of the 
Coulomb energy, $e^{2} / 4 \pi \epsilon l_{B} \simeq 65 B_{\perp}^{1 / 2}$, where $\epsilon \simeq 10 \epsilon_{0}$ is the dielectric constant of AlAs and $l_{B}=\left(\hbar / e B_{\perp}\right)^{1 / 2}$ is the magnetic length (the unit for $B_{\perp}$ is $\mathrm{T}$ and for energy is $\mathrm{K})$ (19). In qualitative agreement with this expectation, the magnitude of the measured $\Delta E_{3,2}$ increases with $B_{\perp}$. Our simple model described by Eq. 1 also explains the shift of resistance peaks from high- $\nu$ to low- $\nu$ with increasing $\theta$. However, the dependence of $\Delta E_{3,2}$ on $B_{\perp}$ is only slightly sublinear rather than proportional to $B_{\perp}^{1 / 2}$. A quantitative understanding of the magnitude of $\Delta E_{3,2}$ and of its dependence on $B_{\perp}$ therefore requires future theoretical work, which includes factors such as the finite layer-thickness of the 2D electrons, the mixing between Landau levels, and disorder.

We then define $\Delta E_{\nu, l}$, in analogy with $\Delta E_{3,2}$, as the difference between bare level energies at the $(\nu, l)$ transition, and plot $\Delta E_{\nu, l}$ in Fig. 3C. We note that all $\Delta E_{\nu, l}$ increase with $B_{\perp}$ and scale approximately as $l$, the number of occupied polarized levels. This scaling, shown in Fig. 3D, is not surprising, because the $l$ polarized levels are the main levels contributing to $\Delta E_{\nu, l}$, although not necessarily with equal weight.

The transition-induced magnetoresistance spikes we have described are apparent in all other samples we have characterized so far and which contain similar carrier densities; these include one sample grown on a GaAs (311)B substrate and five samples grown on (100), all of them $150 \AA$-wide AlAs quantum wells. On the other hand, we do not observe the resistance spikes in highdensity samples with only one subband occupied: In the density range $3.5 \times 10^{11}$ to $5.2 \times 10^{11} \mathrm{~cm}^{-2}$, where we have carefully monitored $R_{x x}$ as a function of tilt angle, we observe that $R_{x x}$ minima do become slightly weaker near coincidence but do not exhibit any additional peaks. At this point, we do not know why peaks appear only at low carrier densities.

We comment briefly on the resistance spikes themselves. We suggest that when the two competing ferromagnetic states acquire the same energy, they separate into domains with opposite magnetizations (within the top Landau level). Extended electron states then dissipate energy by scattering at the ferromagnetic domain walls, which explains why the resistance rises at the transition. Indeed, theoretical studies of the QH liquid at $\nu=1$ (20) hint that magnetic domains induced by a fluctuating Zeeman energy can cause dissipative transport. A similar explanation has also been attributed to negative magnetoresistance in various ferromagnetic materials (21, 22). Nucleation sites for the domains may then be provided by topological quasiparticles akin to skyrmions, which appear when two Landau levels cross (23, 24). This may explain another conspicuous feature of our hysteresis data in Fig. 2C, namely that the magnitude of the transition resistance is systematically higher in the upward sweep than in the downward sweep, independent of the position of the peak with respect to the center of the $R_{x x}$ minimum. This asymmetric trend might be due to the presence of a larger number of skyrmions as the transition is approached from the low- $B$ side, i.e. from the state with the smaller magnetization.

In conclusion, we have demonstrated that a first-order Ising transition, when observed within the quantum Hall regime, has a marked impact on the transport properties of a 2D electron gas. The resistance spikes we observe at the transition can also shed light on the physics of magnetic domains in $2 \mathrm{D}$ electron systems. We have also learned that AlAs quantum wells, which have been scarcely used in the past for measuring magnetotransport, can display phenomena unobserved or unresolved so far in other semiconductor heterostructures. We thus hope that the many structures we have yet to imagine on the basis of this compound, will provide a fertile ground for the observation of new correlated electron phenomena.

\section{REFERENCES}

[1] E. Kisker, in Metallic Magnetism, H. Capellmann, Ed. (Springer-Verlag, Berlin, 1987), pp. 57-108.

[2] V. P. Antropov, B. N. Harmon, A. N. Smirnov, J. Magn. Magn. Mater. 200, 148 (1999).

[3] D. P. Young et al., Nature 397, 412 (1999).

[4] D. Ceperley, Nature 397, 386 (1999).

[5] S. J. Papadakis, E. P. De Poortere, M. Shayegan, Phys. Rev. B 59, R12743 (1999).

[6] S. Koch, R. J. Haug, K. v. Klitzing, M. Razeghi, Phys. Rev. B 47, 4048 (1993).

[7] A. J. Daneshvar et al., Phys. Rev. Lett. 79, 4449 (1997).

[8] T. Jungwirth, S. P. Shukla, L. Smrčka, M. Shayegan, A. H. MacDonald, Phys. Rev. Lett. 81, 2328 (1998).

[9] J. P. Eisenstein, H. L. Stormer, L. Pfeiffer, K. W. West, Phys. Rev. Lett. 62, 1540 (1989).

[10] R. G. Clark, in Localization and Confinement of Electrons in Semiconductors, F. Kuchar, H. Heinrich, G. Bauer, Eds. (Springer-Verlag, Berlin, 1990), pp. $168-182$.

[11] L. W. Engel, S. W. Hwang, T. Sajoto, D. C. Tsui, M. Shayegan, Phys. Rev. B 45, 3418 (1992).

[12] J. Eom et al., Science 289, 2320 (2000).

[13] T. Jungwirth, A. H. MacDonald, Phys. Rev. B, in press.

[14] V. Piazza et al., Nature 402, 638 (1999).

[15] The relevant mass in our measurements is the cyclotron effective mass, which in AlAs (100) is $0.46 m_{e}$, where $m_{e}$ is the bare electron mass [see, for example, T. S. Lay et al., Appl. Phys. Lett. 62, 3121 (1993)]. In AlAs (411)B, on account of geometric arguments, we estimate the cyclotron mass at $0.41 m_{e}$.

[16] H. W. van Kesteren et al., Phys. Rev. Lett. 61, 129 (1988), and references therein.

[17] Data for Figs. 1B, 2, and 3 were collected during different cooldowns. 
[18] In a previous publication (5), we reported an enhanced $\left|g^{*}\right| \simeq 9.0$ for $2 \mathrm{D}$ electrons in AlAs quantum wells, because we chose to combine the effects of both exchange-correlation and Zeeman energies into the effective $g$-factor.

[19] For approximate values of exchange-correlation energies of $2 \mathrm{D}$ electrons in a magnetic field, see, for example, A. H. MacDonald and D. S. Ritchie; [Phys. Rev. B 33, 8336 (1986)].

[20] V. I. Fal'ko, S. V. Iordanskii, Phys. Rev. Lett. 82, 402 (1999).

[21] M. Viret et al, Phys. Rev. Lett. 85, 3962 (2000), and references therein.
[22] P. Schiffer, A. P. Ramirez, W. Bao, S.W. Cheong, Phys. Rev. Lett. 75, 3336 (1995).

[23] T. Jungwirth, I. Mihalek, H. Fertig, D. Lilliehöök, A. H. MacDonald, in preparation.

[24] D. Lilliehöök, Phys. Rev. B 62, 7303 (2000).

[25] We thank Allan MacDonald for illuminating discussions. Our research has been supported by the NSF. We thank Eric Palm and Tim Murphy for their help with parts of our measurements done at the National High Magnetic Field Laboratory, Tallahassee, FL, which is also supported by the NSF. 